\title{
Phonetic analysis in forensic odontology
}

\begin{abstract}
The aim of the study was to analyze the phonetic changes of a patient before and after prosthetic treatment and application of an gingival epithesis which may be a clue for forensic odontology. This proposed case was permanently stabilized by a resin bonded fixed partial denture for prevention of teeth luxation after periodontal and orthodontic treatment. The phonetic changes were tested by a computerized sound superposition programme after the application of the gingival epithesis. The results of these tests showed considerable sound alteration which should be taken into consideration, when conducting forensic phonetic research in the field of forensic odontology.
\end{abstract}

Keywords: forensic odontology, phonetic analysis, dentistry, prosthetic treatment, gingival epithesis

\author{
Volume 6 Issue I - 2018 \\ Kansu Gulay, Aka P Sema,Keskin Yasemin \\ Department of Prosthodontics and Unit of Forensic \\ Odontology, Faculty of Dentistry, Turkey
}

Correspondence: Aka P Sema, Faculty and Head of Forensic Odontology, Department of Prosthodontics and Unit of Forensic Odontology,Ankara University, Ankara, Turkey, Tel 905325136389, Email akasema@gmail.com

Received: October 20, 2017 | Published: January 16, 2018

\section{Introduction}

In general dental practice, a prosthetic replacement is considered to be ideal, when it fulfills esthetic, functional and phonetic demands. It is recommended to make speech tests after establishing occlusion for function and obtaining satisfactory esthetics in dentures. ${ }^{1}$ A prosthetic solution should not be regarded as complete without optimal control of phonetic aspects. For this purpose, some special sounds such as "s", "z", "m", "ch", and "j" are pronounced. The proper pronunciation of these letters shows the correct vertical dimension by using closest speaking space. ${ }^{2,3}$ In advanced periodontal cases where soft tissue recession has occurred a phonetic problem may arise due to evading sounds.

\section{Problem of the study}

The aim of this research was to evaluate the phonetic changes before and after prosthetic treatment and application of a gingival epithesis of such a case indicating sound deficiencies due to tissue destruction.

\section{Materials and methods}

A 43-year-old woman having 22 teeth and destructed bone and supporting tissues, applied for dental treatment (Figure 1). After completion of her periodontal and orthodontic therapy, arch splinting via adhesive partial crown construction were done at the first step of the prosthodontics (Figures 2\&3). Then a flexible gingival epithesis was constructed for esthetic and phonetic requirements, by a onecomponent silicone material (Gingivamoll Detax $\mathrm{GmbH} \& \mathrm{Co} . \mathrm{KG}$, Ettlingen, Germany) which was produced according to the clinical and laboratory technique described by Iselin et al. ${ }^{4}$ (Figure 4 ). Speech records were taken before and after the epithesis insertion, in a standard position where the distance between the 16 BIT stereo microphone and the patient was $40 \mathrm{~cm}$ 'sin a sound proof room. Tests were performed in two steps, where sound characteristics were determined by the pronunciation of letter and text reading. During the speech tests, the letter sounds were classified according to their pronunciationspecialties of basic articulations as sibilants, plosives, fricatives, liquids, glides and nasals which were previously defined by Burnett. ${ }^{5}$

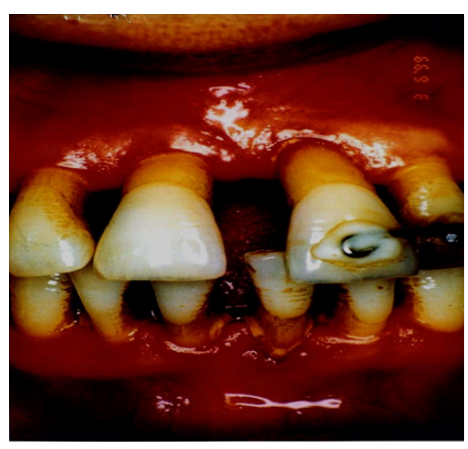

Figure I A case showing advanced destruction of periodontal tissuesthat caused phonetic voice disorders.

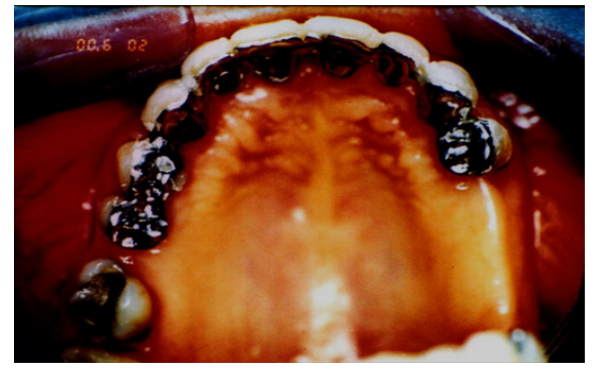

Figure $\mathbf{2}$ The first step of the prosthodontics was the palatinal arch splinting, via adhesive partial crown construction which improved articulation gradually.

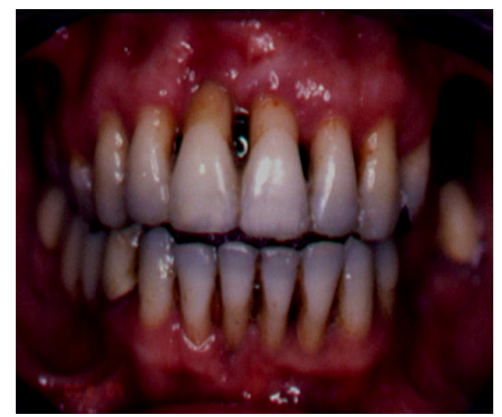

Figure 3 Anterior view of the natural tooth arch which was splinted by an adhesive partial crown construction from the palatinal side. 


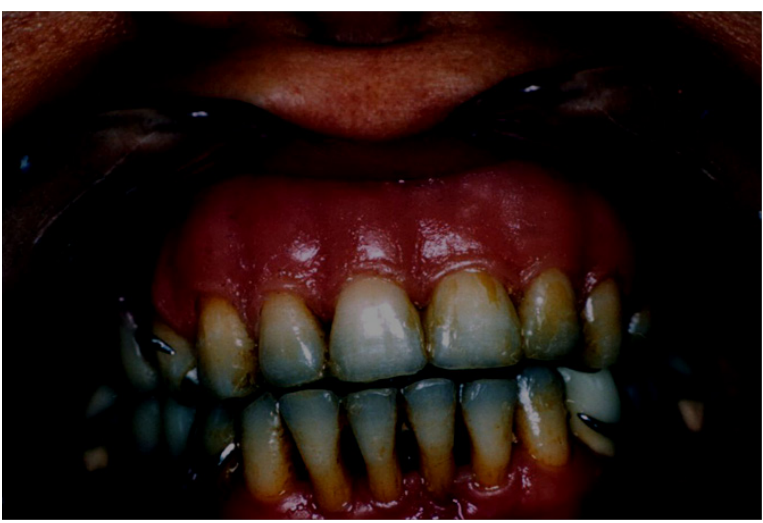

Figure 4 The second step of the prosthodontics was the gingival mask application which prevents excessive air escape through the interproximal areas and alters the phonetic sounds.

In the first step, a specific sound test was accomplished by sounding 28 letters including four sibilants $(\mathrm{j}, \mathrm{s}, \mathrm{s}, \mathrm{z})$, six plosives ( $p, b, t, d, k, g)$, two fricatives (f, v), two liquids $(1, r)$, and two nasal sounds $(m, n)$, which were registered by a computerizedprogramme. Then dimensions of the "voice on set time" of some selected sounds were calculated. In the second step, a text including words with the placement of the chosen consonant sounds as " $\mathrm{j}, \mathrm{l}, \mathrm{n}, \mathrm{s}$ ", and " $\mathrm{t}$ " in the initial, plus an open vowel (a) and a closed vowel (e) were used. These special letters were evaluated from the point of vowel characteristics to establish if these additional vowels are effective on changing the sound dimensions of these consonant letters. Besides, this text sound test, a random speech test was performed disclosing the subjective speech defects, which also showed the words, which are difficult to pronounce. Sound tests of the patient were repeated in periodic check intervals with epithesis in 1, 3 and 6 months. The obtained results were matched with the similar sound records of 10 women age 35-45 having Angle Class I occlusions without any restorations.

The computerized calculation and evaluation process of sound tests are given as follows:

i. The sound records were registered in a computer program called "The Creative Wave Studio" which shows the sound characteristics as sound wave vibration traces.

ii. On the wave vibration traces, the voice on set duration of each sound was measured numerically as byte sizes of waveform display units. Than these data are transferred to an Excelprogramme where the average values were calculated and their graphics were plotted.

iii. The images of the wave traces were amplified five times and exported to a picture processing programme called "i Photo Plus- 4" and "Kodak Photo Imaging" programme.

iv. Each chosen sound character image, which was taken from patient and from the control group, were superposed and compared by arithmetical averages and graphics.

\section{Results}

The results of this research, concerning the application of a gingival epithesis was obtained initially and during the periodical controls (1, 3, 6 months duration). Clinical and computerized sound tests disclosed the fact that, patients articulation improved gradually, when compared to the data obtained from Class I. occlusion unrestored cases $(\mathrm{n}=$
10). According to the sound tests; which were selected in special sound groups, the "sound on set durations (SOD)" of consonants, consonant plus an open and closed vowel articulations were recorded and calculated (Figure 5) (Tables 1-6). The achieved data cleared that, before the epithesis construction and at the time of epithesis insertion the SOD were higher and these data showed a tendency to decrease in 1,3 and 6 month period where the values come closer to the average data of SOD of natural Angle Class I cases.

\section{1. 2. HHHHHHOHHHHHHHHHHHHHHHH+}

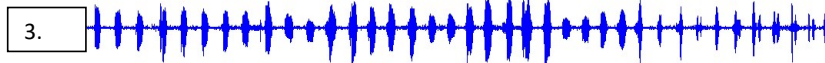

$$
\begin{aligned}
& \text { 1. Withoutepithesis } \\
& \text { 2. Superimposition }
\end{aligned}
$$

Figure 5 Graphics of "sound on set durations" shows considerable alteration of phonetic sounds.

Table I The "voice on set durations" and the average valuesof consonant sound data obtained from thesound records of 10 cases having Angle Class occlusions without any restorations (byte)

\begin{tabular}{llllll}
\hline Case & $\mathbf{s}$ & $\mathbf{t}$ & $\mathbf{f}$ & $\mathbf{l}$ & $\mathbf{n}$ \\
\hline $\mathbf{I}$ & 21014 & 20372 & 15177 & 22224 & 24076 \\
2 & 26392 & 21442 & 21443 & 21442 & 23092 \\
3 & 22319 & 21007 & 14442 & 19694 & 21006 \\
4 & 21522 & 21521 & 18447 & 21522 & 23059 \\
5 & 20205 & 15958 & 16837 & 19504 & 17731 \\
6 & 27123 & 20342 & 18647 & 22037 & 22037 \\
7 & 23900 & 23901 & 19120 & 20714 & 22307 \\
8 & 25768 & 22087 & 20247 & 21480 & 19527 \\
9 & 20862 & 17652 & 17652 & 20862 & 19257 \\
10 & 24779 & 20133 & 15487 & 20133 & 23231 \\
Average & 23388 & 20442 & 17750 & 20961 & 21532 \\
\hline
\end{tabular}

Table 2 The "voice on set durations" of consonant sound data obtained from the patient before and after epithesis insertion showed improvement during six moths (byte)

\begin{tabular}{lllllll}
\hline Epithesis & Date & s & t & f & I & n \\
\hline Before & Insertion & 26115 & 24106 & 20089 & 21626 & 24446 \\
After & Insertion & 32501 & 28168 & 23834 & 21669 & 23739 \\
After & I month & 25583 & 20467 & 20466 & 20466 & 22172 \\
After & 3 month & 28909 & 20649 & 19309 & 21006 & 21029 \\
After & 6 month & 23004 & 20151 & 17865 & 20289 & 20696 \\
\hline
\end{tabular}


Table 3 The "voice on set durations" and the average values of consonant plus open vowel sound data obtained from thesound records of 10 unrestored cases (byte)

\begin{tabular}{llllll}
\hline Case & sa & ta & la & fa & na \\
\hline$I$ & 12390 & 7743 & $1084 \mid$ & 4264 & 4848 \\
2 & 11398 & 7944 & 11208 & 3780 & 5750 \\
3 & 11492 & 7182 & 10149 & 3204 & 3204 \\
4 & 11974 & 6531 & 11346 & 3480 & 4928 \\
5 & 12218 & 6787 & $1037 \mid$ & 3648 & 4600 \\
6 & 11414 & 7610 & 10878 & 3996 & 3760 \\
7 & 10952 & 7740 & 9583 & 3872 & 4558 \\
8 & 11517 & 7678 & 13306 & 3773 & 5355 \\
9 & 9431 & 6736 & 10259 & 3780 & 6552 \\
10 & 10909 & 7273 & 10260 & 3635 & 6045 \\
Average & 11370 & 7322 & 10820 & 3743 & 4960 \\
\hline
\end{tabular}

Table 4 The "voice on set durations" and the average values of consonant plus closed vowel sound data obtained from thesound records of 10 unrestored cases (byte)

\begin{tabular}{llllll}
\hline Case & se & te & fe & le & ne \\
\hline I & 8652 & 4996 & 3672 & 8711 & 2880 \\
2 & 8946 & 4264 & 4264 & 8670 & 3196 \\
3 & 8296 & 4854 & 3204 & 8070 & 3145 \\
4 & 8582 & 4884 & 3480 & 8840 & 2870 \\
5 & 8427 & 4500 & 3522 & 8132 & 3619 \\
6 & 8540 & 3996 & 3066 & 9030 & 3195 \\
7 & 8788 & 3872 & 3780 & 8736 & 3526 \\
8 & 8130 & 3480 & 3930 & 8708 & 3450 \\
9 & 8280 & 3780 & 3396 & 8520 & 3066 \\
10 & 8185 & 3672 & 3927 & 7874 & 2952 \\
Average & 8483 & 4230 & 3624 & 8529 & 3190 \\
\hline
\end{tabular}

Table 5 The "voice on set durations" of consonant plus open vowel sound data obtained from the patient before and after epithesis insertion showed improvement during six moths (byte)

\begin{tabular}{lllllll}
\hline Epithesis & Date & sa & ta & fa & la & na \\
\hline Before & Insertion & 9897 & 7328 & 4020 & 12710 & 5810 \\
After & Insertion & 13112 & 8547 & 4032 & 10560 & 4080 \\
After & I month & 12218 & 6786 & 3927 & 10200 & 3822 \\
After & 3 month & 10594 & 7564 & 3780 & 10780 & 3596 \\
After & 6 month & 10982 & 7306 & 3704 & 10366 & 3623 \\
\hline
\end{tabular}

Table 6 The "voice on set durations" of consonant plus closed vowel sound data obtained from the patient before and after epithesis insertion showed improvement during six moths(byte)

\begin{tabular}{lllllll}
\hline Epithesis & Date & se & te & fe & le & ne \\
\hline Before & Insertion & 8150 & 4914 & 4812 & 9408 & 3224 \\
After & Insertion & 8143 & 4161 & 4780 & 9246 & 3784 \\
After & I month & 8642 & 3498 & 4264 & 8060 & 3283 \\
After & 3 month & 8340 & 3750 & 4230 & 7820 & 3610 \\
After & 6 month & 8910 & 3610 & 4032 & 9252 & 3744 \\
\hline
\end{tabular}

\section{Discussion}

Alterations of the oral cavity can affect speech articulation. ${ }^{6,7}$ Zitzmannet al., ${ }^{8}$ claimed that the excessive air escape through the interproximal areas could cause speech problems. They advocated the construction of a buccal flange for removable overdenture prosthesis to avoid speech disruption and "s" sound deficiencies. These authors claimed that, the extension of the buccal and palatal denture flange establishes of the smile line, labial support and phonetic requirements. ${ }^{9}$ To provide oral hygiene access these researchers also attract attention on creating adequate embrasures without phonetic disturbance..$^{10}$ In this research, these advantages were obtained with the usage of the flange of the epithesis. Witkowski ${ }^{11}$ also recommended the construction of gingival epithesis over implant prosthesis for correcting phonetics, supporting the lip profile, building up esthetics and preventing food impaction for patients having high smile line. In this research, the interproximal spaces were obstructed by the construction of a flexible epithesis, which also served as a foundation for the phonetic zone that is present at the anterior palatal region. This epithesis foundation was used as a buccal flange, which provided speech improvements approved by the speech tests. After the insertion of the epithesis, speech tests data have shown similar results with the control group having Class I. unrestored occlusion.

Speech tests are preferable tests in general dental practice, as it's also reported that, during the speech, patients exhibits seven informative facts which are affected by mandibular movements that directly relates to the vertical and horizontal overlap, lower anterior tooth display, former class of occlusion, vertical dimension, incisal guidance and maximum cusp height. ${ }^{12}$ As an example the prolonged pronunciation of sibilant sounds as " $\mathrm{s}$ " and " $\mathrm{z}$ " when converted into a whistle or swish shows incorrect face height, and obliterated freeway space. ${ }^{1}$ The phonetic control in denture construction phase usually includes this specific "s" sound and sibilant sounds which shows anterior speaking position of mandible and determines the occlusal vertical disruption dimension by establishing the closest speaking space as advocated by Silverman. ${ }^{3}$ Therefore speech tests are accepted as aids for recording vertical dimension where trial bases are used and certain words such as " Mississippi, fifty- five " are pronounced by the patient without striking the rims together. ${ }^{13}$ Besides the labiodental sounds " $f$ and $v$ " which are produced by the contact of the lower lip with the maxillary anterior teeth is a guide for recording vertical dimension and when it is higher the " $\mathrm{v}$ " sound converts to an " $\mathrm{f}$ " sound. ${ }^{14}$ These two letters are called the fricative, which gives the proper position of maxillary incisors, when the incisal edges of these teeth make contact with the lower lip. The plosives such as "p, $b, t$ and $d$ " are characterized by stopping and sudden releasing of the air stream. The nasals "m and n" produced by the occluding lips and passing the sound throughout the nasal cavity. The liquid sounds as "l and $r$ " causes partially escaping of sounds from the tongue laterally and centrally respectively. ${ }^{1}$

In this research the same pronunciation tests were performed after optimizing the articulation spaces of the patient by a gingival epithesis, which closes the interdental spaces. The aforementioned tests showed that, the initial SOD and the sound specialties of the patient changed significantly after the epithesis insertion. Even these data represented similar results with the control group having no restoration in their natural occlusions. As it is seen in that Tables 1-6, the comparison of the special sound groups revealed the following findings:

a. Sibilant sounds: The patients SOD indicated a decrease for consonant "s", and consonant plus open vowel "sa", but a slight increase for the consonant plus closed vowel "se" when 
compared to normal unrestored case average values, which must be due to the direction of the sound towards the epithesis area and a difficult pronunciation of a whistle or swish type closed vowel.

b. Plosive sounds: The plosive "t" sounds showed a tendency to decrease in SOD when compared to normal unrestored case average values in all three categories, by the ease of sudden explosive release of the airstream after the complete occlusion of the tongue to the hard palate.

c. Fricative sounds: The fricatives or the labiodental sounds as " $\mathrm{f}$ " are found to be slightly decreased for all three categories, when compared to normal unrestored case average values as similar to sibilants, which may be due to the forced airstream through a narrow passageway of the epithesis area during pronunciation.

d. Liquid sounds: The liquid or fluid half sounds, showed decrease in all three categories, when compared to normal unrestored case average values due to the difficult pronunciation of the sound, where the tongue apex occludes to the epithesis area, while the sound escapes through the bilateral narrow areas between the tongue and the hard palate.

e. Nasal sounds: The nasals also showed an increase in the consonant plus a closed vowel "ne" categories, when compared to normal unrestored case average values, due to the occlusion of the tongue apex and tongue dorsum to the epithesis area and the hard palate, while the sound partially escapes through the nasal cavity and then partially between the epithesis area and the tip of the tongue while bilateral sides of the tongue contacts to the maxillary teeth.

In this research, the epithesis that was applied over a fixed prosthesis provided a phonetic correction.

\section{Conclusion}

Voice changes may occur after a prosthetic treatment such as, a gingival epithesis that is applied over a resin bonded fixed partial denture, due to the optimal reconstruction of the buccal flanges and phonetic zones. Therefore cases which has undergone prosthetic treatment should be taken into consideration when conducting phonetic research in the field of forensic odontology.

\section{Acknowledgments}

None.

\section{Conflicts of interest}

The author declares there are no conflicts of interest.

\section{References}

1. Sharry JJ. Complete denture prosthodontics. 3rd ed. Mc Graw- Hill, USA, 1974; pp. 130-148.

2. Boucher CO, Hickey JC, Zarb GA. Prosthodontic treatment for edentulous patients. St. Louis: The CV Mosby, USA, 1975; pp. 271-275.

3. Silverman MM. The whistle and swish sound in denture patients. $J$ Prosthet Dent. 1967;17(2):144-148.

4. Iselin W, Meier C, Lufi A, et al. The flexible gingival epithesis. Schweiz Monatsschr Zahnmed. 1990;100(8):967-976.

5. Burnett CA. Mandibular incisor position for English consonant sounds. Int J Prosthodont. 1999;12(3):263-271.

6. Seifert E, Runte C, Riebandt M, et al. Can dental prostheses influence vocal parameters. J Prosthet Dent. 1999;81(5):579-585.

7. Ichikawa T, Komoda J, Horiuchi M, et al. Influence of alterations in the oral environment on speech production. J Oral Rehabil. 1995;22(4):295299.

8. Zitzmann NU, Marinello CP. Treatment plan for restoring the edentulous maxilla with implant supported restorations: Removable overdenture versus fixed partial denture design. J Prosthet Dent. 1999;82(2):188-196.

9. Zitzmann NU, Marinello CP. Clinical and technical aspects of implant supported restorations in the edentulous maxilla: The fixed partial denture design. Int J Prosthodont. 1999;12(4):307-312.

10. Zitzmann NU, Marinello CP. Implant supported removable overdentures in the edentulous maxilla: Clinical and technical aspects. Int J Prosthodont. 1999;12(5):385-390.

11. Witkowski SWJ. Die flexible zahnfleischmaske indikationsbeispiel implantatgetragene brücke. Quintessenz Zahntech. 1991;17:541-557.

12. Pound E. The mandibular movements of speech and their seven related values. J Prosthet Dent. 166;34(9):835-843.

13. Lucia VO. Treatment of the edentulous patient. Quintessence, USA, 1986; pp. 187-193.

14. Lamb DJ. Problems and solutions in complete denture prosthodontics. Quintessence, UK, 1993; pp. 109. 